\title{
Novel Extracorporeal Hemoperfusion System for Hepatic Arterial Infusion of Cisplatin
}

\author{
SHINICHI SO, SHOHEI KOMATSU, ATSUSHI TAKEBE, MASAHIRO KIDO, MOTOFUMI TANAKA, \\ HIROCHIKA TOYAMA, KIMIHIKO UENO and TAKUMI FUKUMOTO \\ Department of Surgery, Division of Hepato-Biliary-Pancreatic Surgery, \\ Kobe University Graduate School of Medicine, Kobe, Japan
}

\begin{abstract}
Background/Aim: Hepatic venous isolation and extracorporeal charcoal hemoperfusion (HVI-CHP) can reduce systemic exposure to hepatic arterial infusion (HAI) chemotherapy. The pre-existing HVI-CHP system has limited effectiveness against high-dose cisplatin; therefore, we designed a new system and evaluated its efficacy in a canine model. Materials and Methods: Cisplatin was administered via HAI under HVI-CHP. HVI-CHP was performed using one charcoal column in group I and two charcoal columns in group II; it was not performed in group III. The plasma cisplatin levels in the systemic circulation and at the column inlet and outlet, and the column extraction rate of were analyzed. Results: The column extraction rates of free and total cisplatin in group II were significantly higher than those in group I. The systemic concentration of free cisplatin was significantly lower in group II than in groups I and III after HAI. No significant differences were observed in cisplatin concentrations in the liver tissue among all groups. Conclusion: A novel HVI-CHP system for HAI of cisplatin was successfully developed.
\end{abstract}

Surgical resection is the optimum procedure for therapy of malignant liver tumors, but the majority of patients with such tumors cannot be subjected to this procedure (1). Although hepatic arterial infusion (HAI) chemotherapy has been widely used to achieve high local drug concentrations and take advantage of the first-pass hepatic clearance of chemotherapeutic agents, its significance remains obscure owing to dose limitation.

Correspondence to: Shohei Komatsu, MD, Ph.D., Department of Surgery, Division of Hepato-Biliary-Pancreatic Surgery, Kobe University Graduate School of Medicine, 7-5-2 Kusunoki-cho, Chuo-ku, Kobe 650-0017, Hyogo, Japan. Tel.: +81 783826302, Fax: +81 783826307, e-mail: komasho8@med.kobe-u.ac.jp

Key Words: Hepatic arterial infusion, cisplatin, extracorporeal charcoal hemoperfusion.
To overcome this situation, we previously developed a novel liver-specific chemotherapy with an extracorporeal drug extraction system, namely percutaneous isolated hepatic perfusion (PIHP) (2-6). High-dose HAI of an antitumor agent was achieved by the combination of hepatic venous isolation and an extracorporeal charcoal hemoperfusion (HVI-CHP) circuit. More than 200 patients with highly advanced hepatocellular carcinoma (HCC) have already received this original therapy during the past two decades and excellent therapeutic outcomes have been reported (7, 8). Meanwhile, our previous experimental study and clinical pilot trial showed that antitumor agents available for PIHP are still limited to doxorubicin and mitomycin $\mathrm{C}$.

Cisplatin is one of the key drugs used against a variety of human cancer types, and the most promising candidate for high-dose infusion therapy owing to its concentrationdependent antitumor effect $(9,10)$. However, cisplatin has a relatively low hepatic extraction; thus, high systemic drug concentrations with resultant systemic toxicity, especially renal dysfunction, occur after its use in HAI. This has prevented significant dose escalation of cisplatin in HAI chemotherapy for liver tumors. We previously evaluated the extraction efficacy of cisplatin after HAI using a single charcoal column in HVI-CHP with our PIHP system (11). The efficacy of our system was low, thereby restricting its clinical application. The establishment of a new PIHP system fully optimized for HAI of cisplatin can dramatically improve the therapeutic spectrum of cisplatin against liver tumors. Therefore, we designed a new drug-extraction system with HVI-CHP using two columns, in which the column was replaced after $10 \mathrm{~min}$ from the initiation of HAI. The aim of the present study was to evaluate the efficacy of our newly-developed PIHP system for HAI of cisplatin to the liver, with special focus on pharmacokinetics and renal function in an experimental model.

\section{Materials and Methods}

Animal preparation. A total of 20 one-year-old female Beagles (Kitayama Labes Co. Ltd., Yamaguchi, Japan), weighing between 7.9 
and $10.7 \mathrm{~kg}$ were used in this study. They were fed a standard diet and observed for a week to ensure good health. Food was withheld for $12 \mathrm{~h}$ preoperatively. At the end of the study, the dogs were euthanized with a lethal dose of potassium chloride. The study protocol was approved by the Institutional Animal Care and Use Committee of Shin Nippon Laboratories (Approval No: IACUC20130219-3-01). The study was carried out according to Shin Nippon Biomedical Laboratories animal experimentation regulation.

Experiment details and treatment groups. All dogs received highdose HAI of cisplatin ( $4 \mathrm{mg} / \mathrm{kg}$; IA-call ${ }^{\mathrm{TM}}$, Nippon Kayaku Co. Ltd., Tokyo, Japan) over $20 \mathrm{~min}$, and they were divided into three groups according to the procedure used for HVI-CHP (Figure 1). Briefly, HVI-CHP was performed using one charcoal column in group I and two charcoal columns in group II; it was not performed in group III.

Group I: HAI with HVI-CHP using one charcoal column: Group I consisted of four dogs that received HAI with HVI-CHP using one charcoal column for $20 \mathrm{~min}$. Blood samples were collected at 5, 10, 15 , and $20 \mathrm{~min}$ after the initiation of HAI. Liver tissues were sampled soon after the completion of HAI (20 min) and $24 \mathrm{~h}$ later (day 1).

Group II: HAI with HVI-CHP using 2 charcoal columns. Group II consisted of eight dogs that received HAI with HVI-CHP using 2 charcoal columns for $20 \mathrm{~min}$ (10 $\mathrm{min}$ in each). In this group, the charcoal column was replaced with a new one 10 min after the initiation of HAI. Among four of the eight dogs, the liver tissues were sampled immediately after the completion of HAI (20 min) and $24 \mathrm{~h}$ later (day 1). In the remaining four dogs, the liver and kidney tissues were sampled on postoperative day (POD) 7. Blood samples were collected at 5, 10, 15, $20 \mathrm{~min}$, and $24 \mathrm{~h}$ (day 1) from eight dogs, and at the POD 3, 5, and 7 from four dogs.

Group III: HAI without HVI-CHP. Group III consisted of eight dogs that received HAI alone without HVI-CHP. The timings of the blood as well as liver and kidney tissue sampling were similar to those for group II.

The pharmacokinetic analysis of the extraction rate (ER) of the CHP column and changes in plasma biomarkers were analyzed for all three groups, whereas for the long-term outcome, the renal function was analyzed in groups II and III.

Experimental procedure. The dogs were anesthetized by the intravenous administration of sodium pentobarbital $(25 \mathrm{mg} / \mathrm{kg}$ body weight) and pancuronium bromide $(0.1 \mathrm{mg} / \mathrm{kg}$ body weight). After endotracheal intubation, the dogs were mechanically ventilated throughout the experiment. A catheter (6-Fr) was inserted into the right external jugular vein, and lactated Ringer's solution was administered to maintain blood pressure during the procedure. An arterial line was placed into the right carotid artery for both blood pressure monitoring and blood sampling.

For HVI-CHP, a blood-delivering catheter (16-Fr) was placed in the right external jugular vein. Laparotomy was then performed with a midline incision from the xiphoid to the pubis. A blood return catheter (14-Fr) was placed in the retrohepatic inferior vena cava (IVC) through the right femoral vein to drain the hepatic effluent. An infusion catheter (4-Fr) was introduced into the proper hepatic artery via the gastroduodenal artery. The diaphragm just above the IVC was incised in preparation for the clamping of the suprahepatic IVC. The infrahepatic IVC was exposed in the renal vein branches. Following thoracotomy, HVI-CHP was achieved with concomitant vascular clamps placed at the suprahepatic and infrahepatic IVC. Both blood delivering and return catheters were connected to an extracorporeal unit consisting of a centrifugal pump (Biopump-50: Medtronic BioMedicus, Inc., Eden Prairie, MN, USA) and a charcoal drug extraction column (Hemosorba CHS-350 column; Asahi Kasei Medical Co., Ltd., Tokyo, Japan, Figure 2). Once the system was established, the blood was anticoagulated by heparin administration (100 units $/ \mathrm{kg}$ ). Subsequently, a 20 min HAI of cisplatin at a dose of $4 \mathrm{mg} / \mathrm{kg}$ was initiated with a syringe infusion pump (Terufusion Model STC-523; Terumo Co., Ltd.; Tokyo, Japan), followed by HVI-CHP, which was performed following the manufacturer's protocols.

Measurement of cisplatin concentration in plasma. During HVI-CHP, the blood samples were collected in heparinized tubes from the preCHP column line (hepatic venous blood), post-CHP column line, and the left carotid artery (systemic blood). They were placed immediately on ice, centrifuged at $1,000 \times g$ at $4^{\circ} \mathrm{C}$ for $10 \mathrm{~min}$, and then the plasma was removed. The plasma samples were analyzed directly in order to determine the free and total cisplatin concentrations. For free cisplatin measurement, approximately $1 \mathrm{ml}$ of plasma was transferred immediately to a YMT ultrafiltration membrane cone (Centrifree Micropartition Devices; Amicon Inc., Beverly, MA, USA) and centrifuged at $1,000 \times g$ at $4^{\circ} \mathrm{C}$ for $20 \mathrm{~min}$. The ultrafiltrate fraction was used for determining the free cisplatin concentration. A flameless atomic absorption spectrometric method was used to measure the cisplatin concentration as described previously (12).

Measurement of cisplatin level in liver tissue. The concentration of cisplatin in the liver tissues was calculated during the pre- and postoperative periods every $5 \mathrm{~min}$ in the three groups. The liver tissue sample obtained after sectioning and nitric acid were mixed in 1:3 weight ratio, and thermolysis was performed using an ETHOS TC microwave digestion system (Shin Nippon Biomedical Laboratories, Ltd., Pharmacokinetics and Bioanalysis Center, Wakayama, Japan). The cisplatin concentration of the decomposition products was calculated. The time program of ETHOS TC was 0.00-5.00: $0 \rightarrow 600 \mathrm{~W}, 90^{\circ} \mathrm{C} ; 5.01-25.00: 700 \mathrm{~W}$, $90^{\circ} \mathrm{C}$; and 25.01-45.00: $700 \rightarrow 0 \mathrm{~W}, 0^{\circ} \mathrm{C}$ ). The aliquot volume of the decomposition products was weighed, and the density of the decomposition products was calculated. The decomposition products were pre-treated, and the mixtures were injected into an inductively coupled plasma-mass spectrometer (Agilent 7700 Series; Shin Nippon Biomedical Laboratories, Ltd., Pharmacokinetics and Bioanalysis Center, Wakayama, Japan).

Pharmacokinetic and pathological evaluation. The blood flow rate in the hepatic vein was monitored continuously with an electromagnetic flow probe (Bioprobe TX40; Medtronic Bio-Medicus, Inc., Eden Prairie, MN, USA) placed in the pre-CHP column circuit line. The drug-clearance efficacy of HVI-CHP was defined as the the amount of drug removed as a percentage of the amount of drug administered. The drug ER of the CHP column at each sampling time was calculated as follows: $\mathrm{ER}(\%)=(\mathrm{ICa}-\mathrm{OCa}) / \mathrm{ICa} \times 100$, where $\mathrm{ICa}$ was the inlet drug concentration $(\mu \mathrm{g} / \mathrm{ml})$ at sampling time a; OCa was the outlet drug concentration $(\mu \mathrm{g} / \mathrm{ml})$ at sampling time a. To investigate the effect of cisplatin on the renal parenchyma, the renal tissues of the dogs in groups II and III were evaluated from a pathological point of view on POD 7.

Statistical analyses. To compare the continuous data between the groups, the data were tested for normal distributions and an 
Group I $(\mathrm{n}=4)$

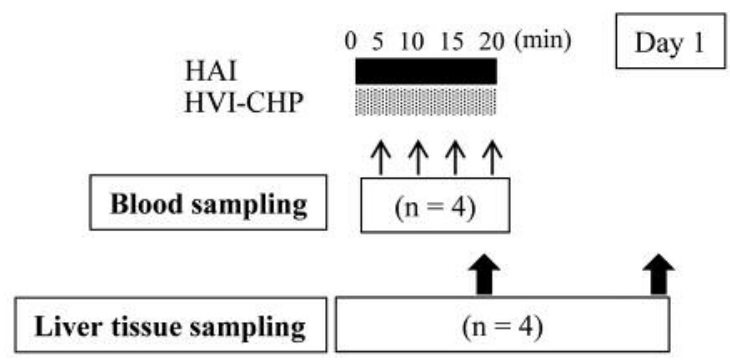

Group II $(\mathrm{n}=8)$

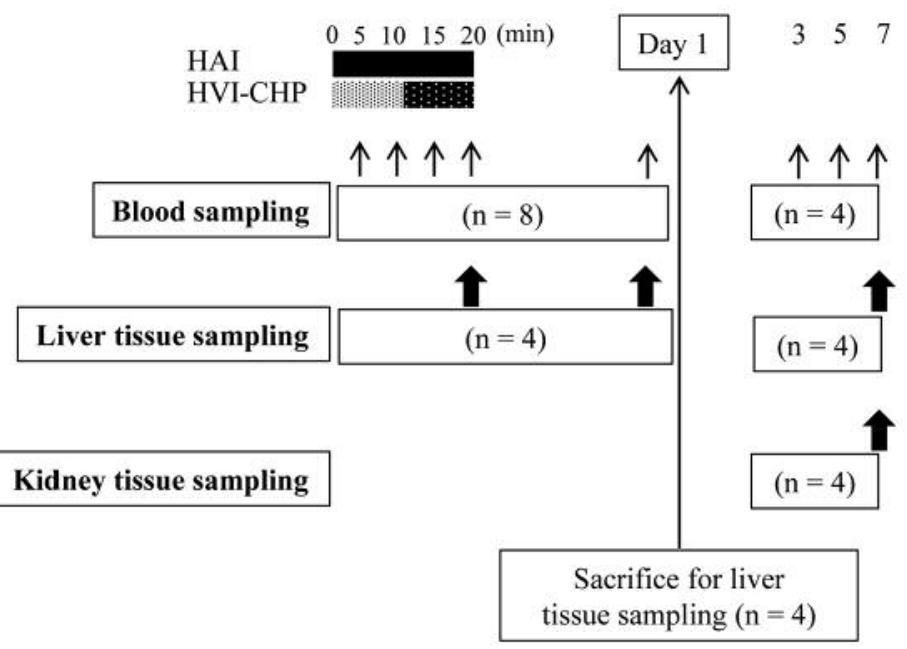

Group III $(\mathrm{n}=8)$

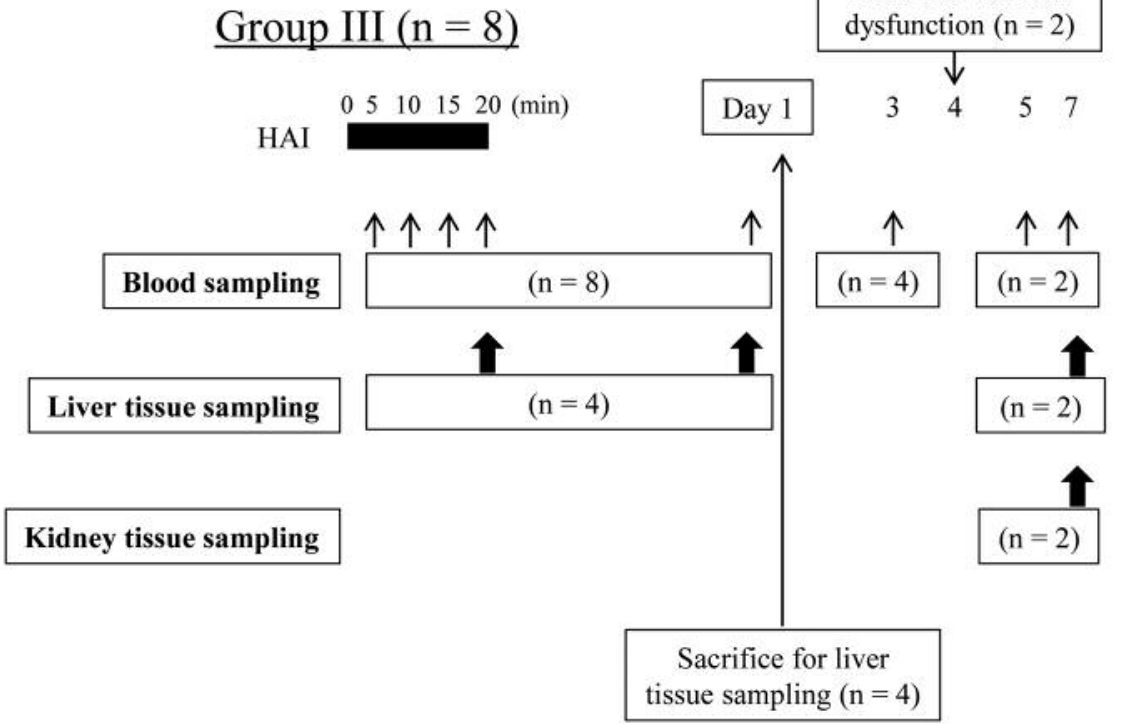

Figure 1. Outline of the study and experimental models used. HAI: Hepatic arterial infusion; HVI-CHP: hepatic venous isolation and extracorporeal charcoal hemoperfusion.

unpaired $t$-test was performed when appropriate; otherwise, the Mann-Whitney $U$-test was used. The categorical data were analyzed using the Chi-squared test or Fisher's exact test, as appropriate. $p$-Values of less than 0.05 were considered statistically significant. All statistical analyses were performed using the JMP 10 statistical package (SAS Institute Cary, NC, USA).

\section{Results}

Plasma profile of cisplatin concentration during the first hepatic pass. The pharmacokinetics of cisplatin during the first pass through the liver parenchyma is shown in Figure 3. The free and total cisplatin concentrations over time at the inlet of the CHP column (before the charcoal column) in groups I and II are shown in Figure 3A. The mean percentage of free to total cisplatin concentration at 5, 10, 15 , and $20 \mathrm{~min}$ after the initiation of HAI is shown in Figure 3B. The percentage of free to total cisplatin concentration was maintained at about $90 \%$ at all specified time points in both groups I and II during HAI with HVICHP. 

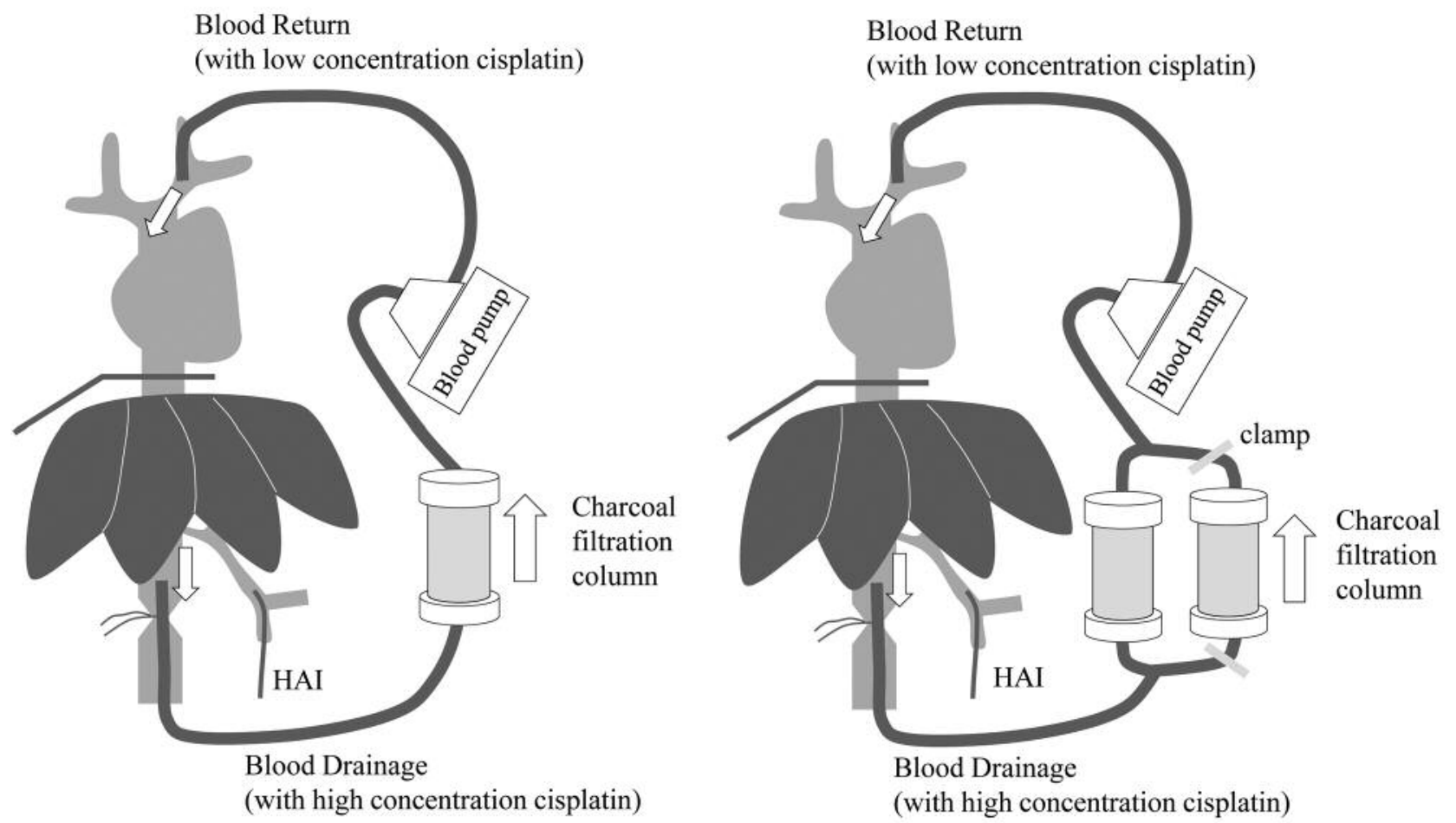

Figure 2. Schematic of the experimental model of hepatic venous isolation and charcoal hemoperfusion. Cisplatin (4 mg/kg) was infused for $20 \mathrm{~min}$ via hepatic arterial infusion. HAI: Hepatic arterial infusion.

A

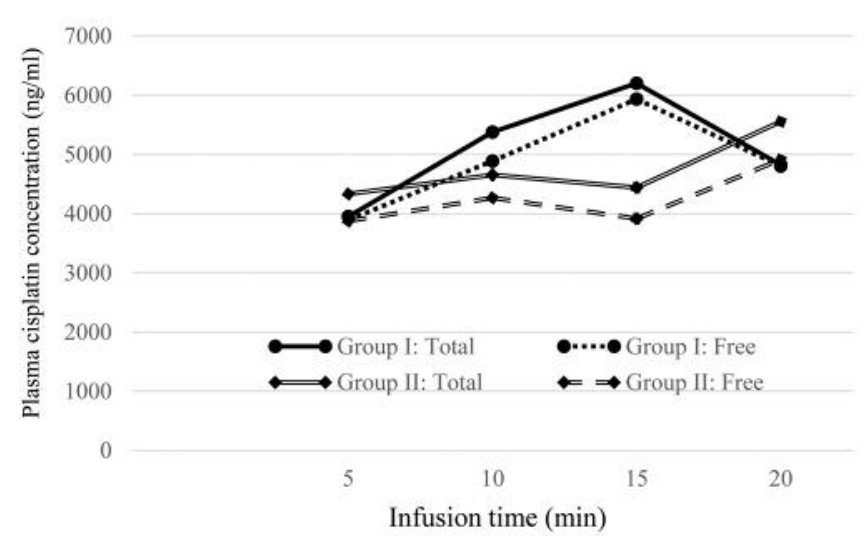

B

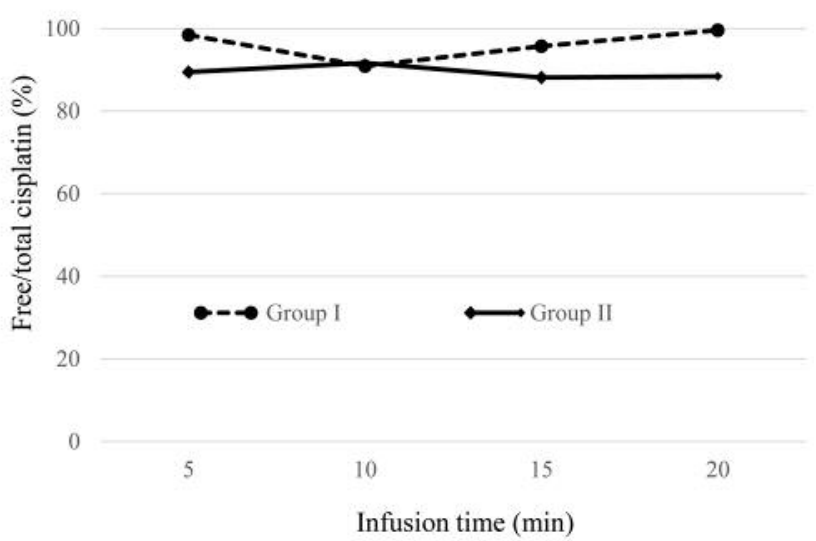

Figure 3. The pharmacokinetics of cisplatin during the first pass through the liver parenchyma in groups I and II. A: Free and total plasma cisplatin concentrations over time at the inlet of the hepatic venous isolation and charcoal hemoperfusion column. B: The mean percentage of free to total cisplatin concentration.

Extraction rates of cisplatin with single and double HVICHP. The mean ERs for free and total cisplatin in group I (HVI-CHP using one column) and group II (HVI-CHP using two columns) are shown in Figure 4. In group I, a high ER level was maintained for both total and free cisplatin during the first half of HAI (more than $80 \%$ in the first 5 and 10 min); however, it gradually, but significantly decreased with time (around $50 \%$ at $20 \mathrm{~min}$ ). Significantly higher ERs were seen in group II even at 15 and $20 \mathrm{~min}$, when compared to those in group I (total cisplatin: $p=0.0084$ at $15 \mathrm{~min}$, and 
A

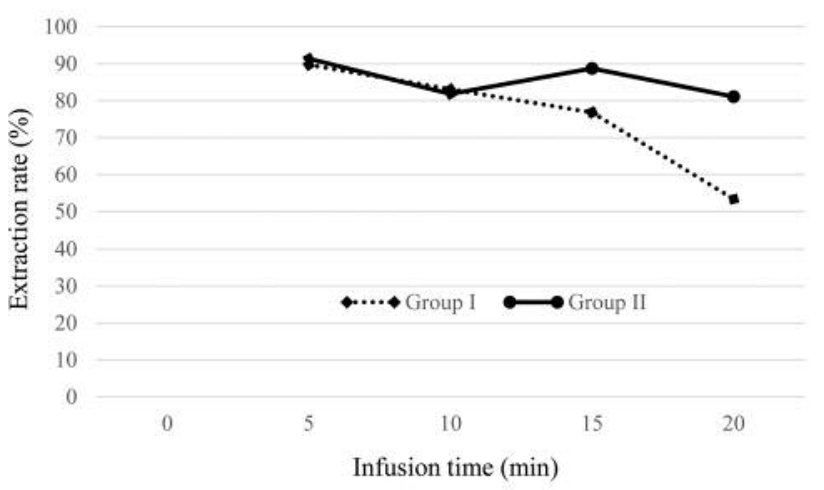

B

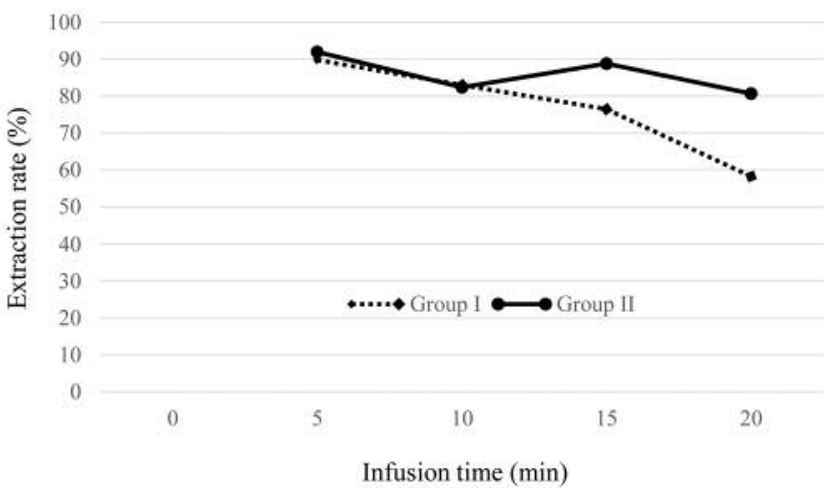

Figure 4. The mean extraction rate of cisplatin in groups I and II. A: Total cisplatin, and B: free cisplatin.
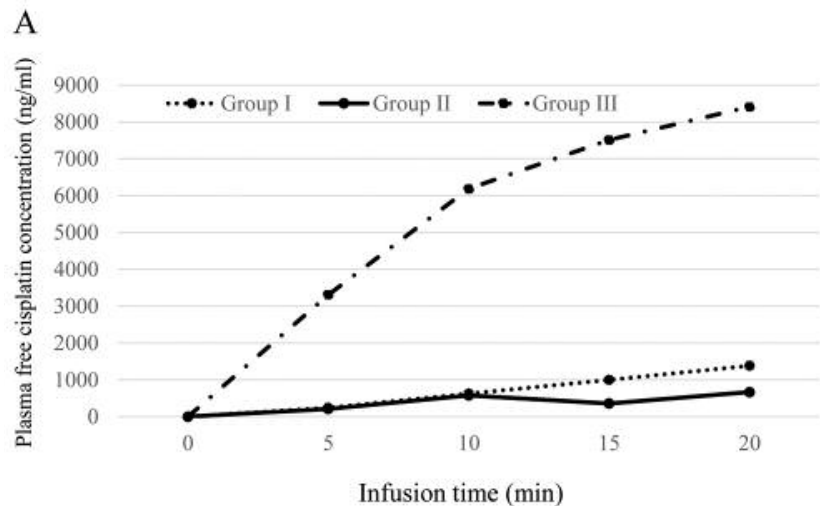

B

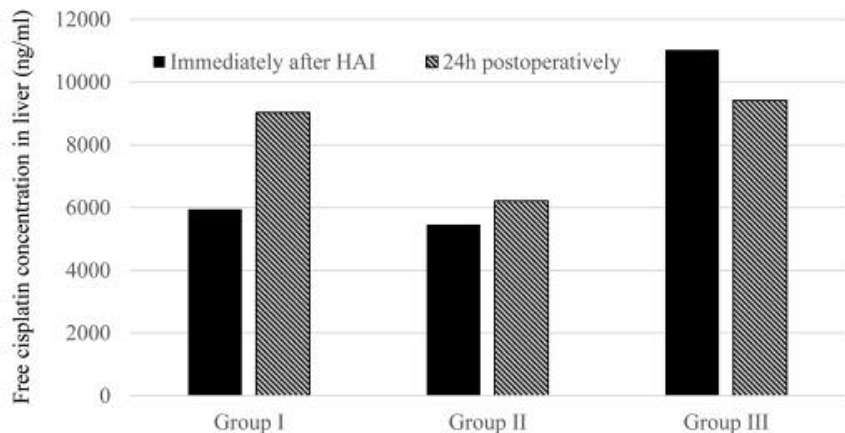

Figure 5. A: Median systemic blood concentration of free cisplatin over time in all groups. B: Concentration of free cisplatin in the liver tissue immediately after hepatic arterial infusion and $24 H$ postoperatively for all groups. HAI: Hepatic arterial infusion.

$p=0.0439$ at $20 \mathrm{~min}$; free cisplatin: $p=0.0034$ at $15 \mathrm{~min}$, and $p=0.0291$ at $20 \mathrm{~min}$ ).

Cisplatin concentrations in systemic blood and liver tissue. The median systemic concentration of free cisplatin in all three groups is shown in Figure 5a. The systemic concentrations of free cisplatin in group I and group II were significantly lower than those in group III at all points after HAI $(p<0.001)$. Moreover, the median systemic free cisplatin concentration in group II was significantly lower than that in group I at 15 and $20 \mathrm{~min}$ after HAI initiation (Figure 5A; $p=0.0002$ at $15 \mathrm{~min}$, and $p=0.0025$ at $20 \mathrm{~min}$ ). The systemic concentration of free cisplatin in group I gradually increased with time despite continuous drug extraction.

In contrast, there were no significant differences in the concentrations of cisplatin in the liver of all three groups, both immediately after HAI and $24 \mathrm{~h}$ postoperatively
( $p=0.179$ and 0.91 , respectively; Figure 5B). In addition, there were no significant differences with regard to the concentration of cisplatin in the liver tissue between groups I and II, both immediately after HAI and $24 \mathrm{~h}$ postoperatively ( $p=0.896$ and 0.521 , respectively; Figure 5B). In all groups, a high level of free cisplatin was maintained in the liver tissue immediately after HAI and $24 \mathrm{~h}$ postoperatively.

Adverse events after HAI. The chronological changes of plasma creatinine $(\mathrm{Cr})$ level after operation in groups II and III are shown in Figure 6. Among the four dogs in group II, no renal dysfunctions were observed in three, and moderate renal dysfunction was observed in one (plasma $\mathrm{Cr}$ level increased from 0.71 to 1.46 on POD7). Of the four dogs in group III, two died due to severe renal dysfunction on POD4. The mean plasma Cr level of group II on POD7 was significantly lower than that of group III (0.80 vs. 8.04, $p=0.004)$. 


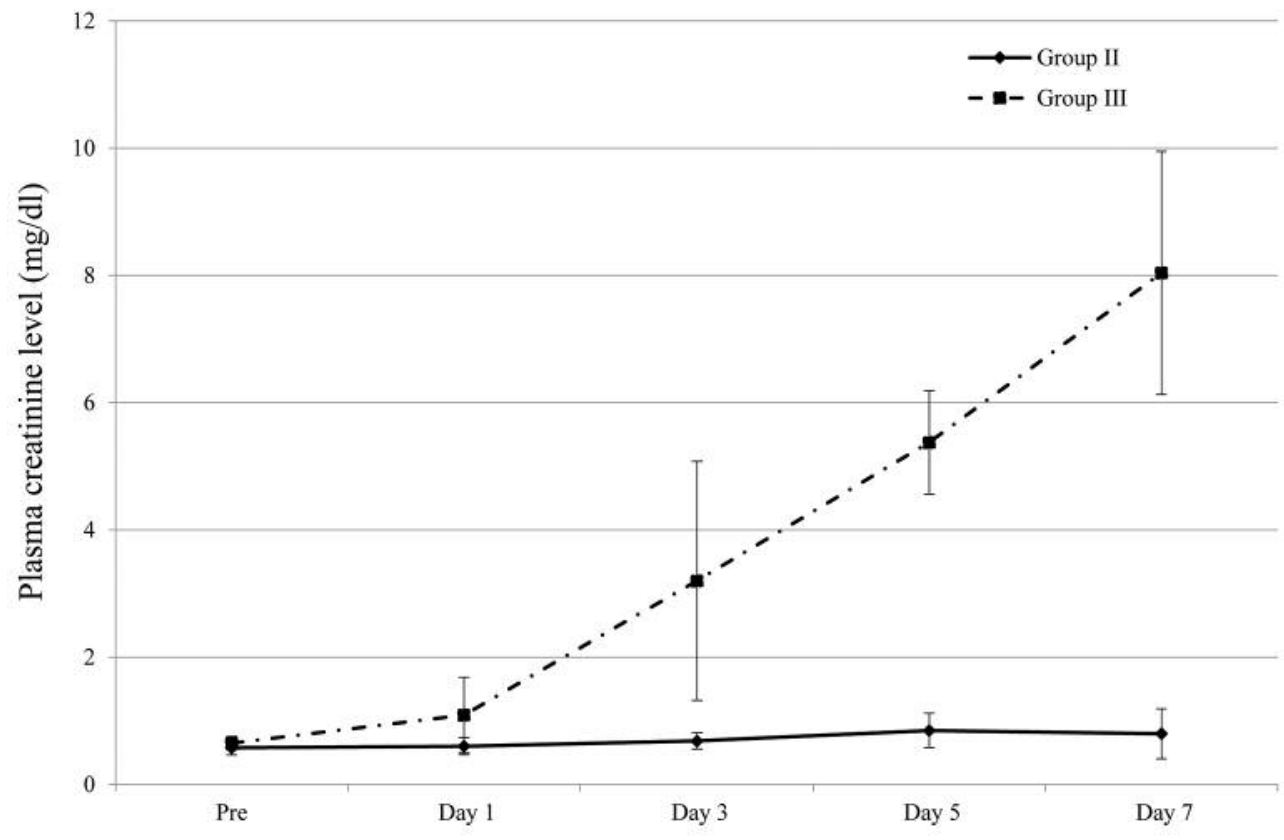

Figure 6. Plasma creatinine level over time after infusion in groups II and III.

The mean concentrations of cisplatin at the renal parenchyma were $666 \mathrm{ng} / \mathrm{g}$ in group II and $6925 \mathrm{ng} / \mathrm{g}$ in group III $(p=0.0062)$. The results of pathological analysis of the kidneys in groups II and III on POD7 are shown in Figure 7. No significant changes were seen in three out of the four dogs in group II, and only a mild dilation of distal renal tubules was seen in the remaining dog. Severe degeneration and necrosis of renal tubules was seen in all four dogs in group III.

\section{Discussion}

The liver is one of the most appropriate targets for regional chemotherapy with isolated blood circulation because of its anatomical peculiarity $(13,14)$. Several preliminary studies with animal models and clinical pilot trials on isolated liver chemotherapy for malignant liver tumors have been reported since the 1960s $(15,16)$. Although a notable antitumor response for isolated chemotherapy with open-abdomen method in advanced liver tumors was reported recently (14), the complexity of the procedure has precluded its widespread clinical implementation (17). Our PIHP system does not require a major operative procedure, thereby dramatically reducing surgical invasiveness during the isolated liver circulation, and it can be used repeatedly in contrast to the open approach (2-6). In 1998, our first report on the long-term results of PIHP in 28 patients with advanced-stage HCC treated with a doxorubicin-based regimen showed that the response rate was $63 \%$. The overall survival rate was $67.5 \%$ and $39.7 \%$ at 1 and 5 years, respectively (5). Since then, more than 200 patients have received high concentrations of doxorubicin and mitomycin C via PIHP with excellent outcomes (8).

The urgent issue which needs to be addressed regarding PIHP is that cases refractory to existing regimens (doxorubicin and mitomycin C) are unable to receive further effective treatments. Although cisplatin is the most promising candidate for PIHP, our initial studies on extracorporeal drug extraction in animal models failed to demonstrate the feasibility of cisplatin due to its severe toxicity $(11,18)$. The present study reconfirmed the results of our previous study (11): the ER of cisplatin with HVICHP using one charcoal column decreased significantly with time (Figure 4). This decline in ER during HAI perhaps depended on the capacity of the drug-filtration system, indicating that the single-column system should not be used for clinical application. Therefore, we designed a new drugextraction system with HVI-CHP using two columns, in which the column was replaced after $10 \mathrm{~min}$ from the initiation of HAI. This successfully overcame the performance deterioration of the single-column system. The median systemic blood concentration of free cisplatin 15 and 20 min after HAI initiation in the group treated using the two-column system was significantly lower than that of those treated using the single-column system (Figure 5A). Furthermore, the analysis of hepatic cisplatin level immediately after HAI and $24 \mathrm{~h}$ postoperatively (Figure 5B) demonstrated that a sufficient cisplatin level was maintained 
A

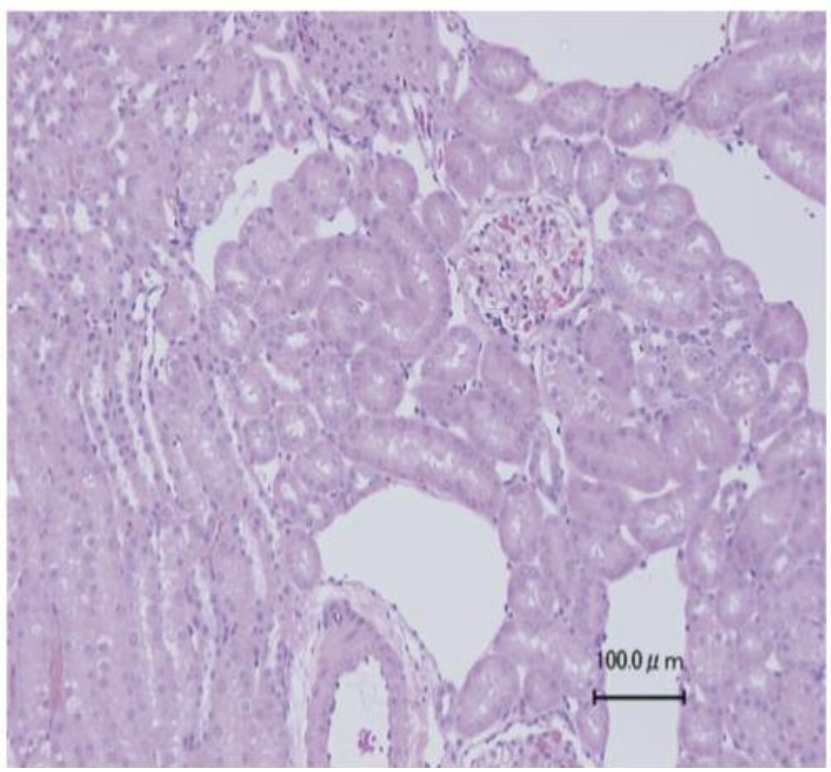

B

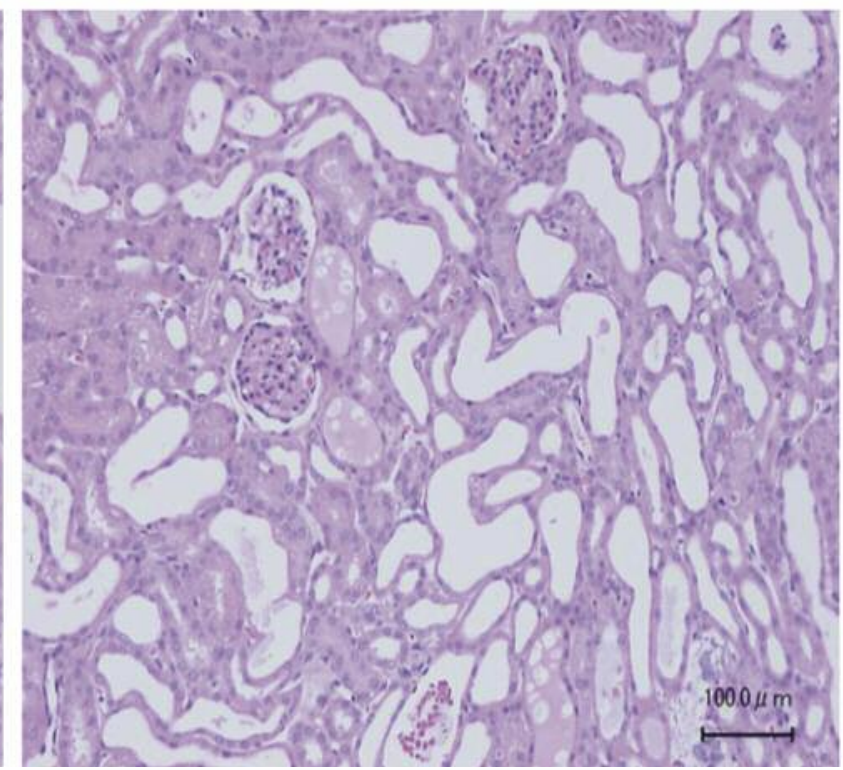

Figure 7. Pathological findings of the kidneys of group II (A) and group III (B). Hematoxylin and eosin staining.

using the two-column system compared with the singlecolumn one. This favorable distribution of systemic cisplatin concentration was due to the continuous maintenance of high ER with HVI-CHP using two columns. In addition, considering the equivalent hepatic concentration of cisplatin in group II (two-column) compared with that in groups I and III (Figure 5B), there seemed to be little influence associated with cisplatin re-entry into the liver during HAI. With regard to the renal function, three out of four dogs in group II had normal renal function on POD7, whereas the remaining dog had mild renal dysfunction. The renal function of dogs in group II was significantly better than that of dogs in group III (Figure 6), indicating the feasibility and effectiveness of the new system. This improved method is a substantial achievement and enables maintenance of a high cisplatin ER during HAI.

It has been reported that more than $90 \%$ of injected free cisplatin is transformed to protein-bound cisplatin within an hour (19). Protein-bound cisplatin was reported to lose both antitumor effect and multi-organ toxicity (19). Interestingly, in this study, the pharmacokinetics of HAI with cisplatin revealed that immediately after the hepatic first pass, almost no cisplatin was bound, and a high concentration of free cisplatin was maintained for $20 \mathrm{~min}$ (Figure 3). This pharmacokinetics seems quite ideal for HAI with cisplatin and is a novel finding in our experimental model.

The feasibility and effectiveness of the PIHP system for clinical use have already been demonstrated by our numerous data $(5,7,8)$. Furthermore, an improved method using a lethal dose of cisplatin without any severe toxicity was demonstrated in basic research in the present study. These findings may represent a great step towards the clinical application of PIHP using cisplatin for patients with refractory advanced HCC. If the present PIHP technique using cisplatin can be incorporated into treatment, it may have great potential not only with second-line antitumor drugs for advanced HCC, but also for other primary and secondary malignant liver tumors, including intrahepatic cholangiocarcinoma and metastatic liver tumors. If high-dose cisplatin can be applied clinically for these tumors using this technique, it may potentially shorten the treatment duration and reduce associated toxicities, thereby providing great impact on treatment strategy.

For the future clinical application of high-dose cisplatin in this way, many existing problems need to be solved. Preliminary studies have shown that the cisplatin concentration in the cytoplasm of hepatocytes should be analyzed using a different method such as synchrotron radiation X-ray fluorescence microscopy (20) because the residual plasma cisplatin in tissue samples potentially affects the results for the cisplatin concentration measured in the liver parenchyma. From a clinical point of view, the development of a more sophisticated type of hemofiltration column optimized for use in PIHP is strongly desired because the charcoal-based hemofiltration column used in this study is not optimized to extract small-size molecules such as cisplatin efficiently. In addition, further efforts to 
reduce the plasma cisplatin concentration are required for the establishment of a safer system. The combined use of sodium thiosulphate $(11,21,22)$ and a hemodialysis system can potentially reduce the plasma cisplatin level after PIHP. These combinations seem to have a synergistic effect and may be an evolving treatment option.

In conclusion, improved hepatic isolated chemotherapy with a high-dose HAI of cisplatin using a two-column HVI-CHP system in an animal model has been successfully developed without complications. This therapeutic option may be an innovative approach for patients with primary or secondary liver tumors, which currently has no indicated treatment.

\section{Conflicts of Interest}

The Authors declare that they have no competing interests in regard to this study.

\section{References}

1 Kim WR, Gores GJ, Benson JT, Therneau TM and Melton LJ, 3rd: Mortality and hospital utilization for hepatocellular carcinoma in the United States. Gastroenterology 129: 486-493, 2005.

$2 \mathrm{Ku}$ Y, Saitoh M, Nishiyama H, Fujiwara S, Iwasaki T, Tominaga M, Maekawa Y, Ohyanagi $\mathrm{H}$ and Saitoh Y: Extracorporeal removal of anticancer drugs in hepatic artery infusion: the effect of direct hemoperfusion combined with venovenous bypass. Surgery 107: 273-281, 1990.

$3 \mathrm{Ku}$ Y, Fukumoto T, Iwasaki T, Tominaga M, Samizo M, Nishida T, Kuroda Y, Hirota S, Sako M, Obara H and Saitoh Y: Clinical pilot study on high-dose intraarterial chemotherapy with direct hemoperfusion under hepatic venous isolation in patients with advanced hepatocellular carcinoma. Surgery 117: 510-519, 1995.

$4 \mathrm{Ku}$ Y, Fukumoto T, Tominaga M, Iwasaki T, Maeda I, Kusunoki N, Obara H, Sako M, Suzuki Y, Kuroda Y and Saitoh Y: Single catheter technique of hepatic venous isolation and extracorporeal charcoal hemoperfusion for malignant liver tumors. Am J Surg 173: 103-109, 1997.

$5 \mathrm{Ku} \mathrm{Y,} \mathrm{Iwasaki} \mathrm{T,} \mathrm{Fukumoto} \mathrm{T,} \mathrm{Tominaga} \mathrm{M,} \mathrm{Muramatsu} \mathrm{S,}$ Kusunoki N, Sugimoto T, Suzuki Y, Kuroda Y, Saitoh Y, Sako M, Matsumoto S, Hirota S and Obara H: Induction of long-term remission in advanced hepatocellular carcinoma with percutaneous isolated liver chemoperfusion. Ann Surg 227: 519$526,1998$.

6 Iwasaki T, Ku Y, Kusunoki N, Tominaga M, Fukumoto T, Muramatsu S and Kuroda Y: Regional pharmacokinetics of doxorubicin following hepatic arterial and portal venous administration: evaluation with hepatic venous isolation and charcoal hemoperfusion. Cancer Res 58: 3339-3343, 1998.

$7 \mathrm{Ku}$ Y, Iwasaki T, Tominaga M, Fukumoto T, Takahashi T, Kido M, Ogata S, Takahashi M, Kuroda Y, Matsumoto S and Obara H: Reductive surgery plus percutaneous isolated hepatic perfusion for multiple advanced hepatocellular carcinoma. Ann Surg 239: 53-60, 2004.

8 Fukumoto T, Tominaga M, Kido M, Takebe A, Tanaka M, Kuramitsu K, Matsumoto I, Ajiki T and Ku Y: Long-term outcomes and prognostic factors with reductive hepatectomy and sequential percutaneous isolated hepatic perfusion for multiple bilobar hepatocellular carcinoma. Ann Surg Oncol 21: 971-978, 2014.

9 Frezza M, Hindo S, Chen D, Davenport A, Schmitt S, Tomco D and Dou QP: Novel metals and metal complexes as platforms for cancer therapy. Curr Pharm Des 16: 1813-1825, 2010.

10 Florea AM and Busselberg D: Cisplatin as an anti-tumor drug: cellular mechanisms of activity, drug resistance and induced side effects. Cancers 3: 1351-1371, 2011.

11 Kusunoki N, Ku Y, Tominaga M, Iwasaki T, Fukumoto T, Muramatsu S, Sugimoto T, Tsuchida S, Takamatsu M, Suzuki Y and Kuroda Y: Effect of sodium thiosulfate on cisplatin removal with complete hepatic venous isolation and extracorporeal charcoal hemoperfusion: A pharmacokinetic evaluation. Ann Surg Oncol 8: 449-457, 2001.

12 LeRoy AF, Wehling ML, Sponseller HL, Friauf WS, Solomon RE, Dedrick RL, Litterst CL, Gram TE, Guarino AM and Becker DA: Analysis of platinum in biological materials by flameless atomic absorption spectrophotometry. Biochem Med 18: 184-191, 1977.

13 Kajanti M, Rissanen P, Virkkunen P, Franssila K and Mantyla $\mathrm{M}$ : Regional intra-arterial infusion of cisplatin in primary hepatocellular carcinoma. A phase II study. Cancer 58: 23862388, 1986.

14 Magge D, Choudry HA, Zeh HJ, 3rd, Cunningham DE, Steel J, Holtzman MP, Jones HL, Pingpank JF, Bartlett DL and Zureikat AH: Outcome analysis of a decade-long experience of isolated hepatic perfusion for unresectable liver metastases at a single institution. Ann Surg 259: 953-959, 2014.

15 Ausman RK: Development of a technic for isolated perfusion of the liver. N Y State J Med 61: 3993-3997, 1961.

16 Healey JE Jr.: A technique for isolation and perfusion of the intact canine liver. Am J Gastroenterol 35: 9-22, 1961.

17 Schaefer CE: Regional perfusion utilizing an extracorporeal circuit: a new technique for the chemotherapy of cancer. J Kans Med Soc 63: 238-247, 1962.

$18 \mathrm{Ku}$ Y, Kusunoki N, Kitagawa T, Maeda I, Fukumoto T, Iwasaki T, Tominaga M, Suzuki Y, Kuroda Y, Tanigawara Y and Saitoh Y: Pharmacokinetics of adriamycin and cisplatin for anhepatic chemotherapy during liver transplantation. Cancer Chemother Pharmacol 40: 457-462, 1997.

19 Ma J, Verweij J, Kolker HJ, van Ingen HE, Stoter G and Schellens JH: Pharmacokinetic-dynamic relationship of cisplatin in vitro: Simulation of an i.v. bolus and $3 \mathrm{~h}$ and $20 \mathrm{~h}$ infusion. Br J Cancer 69: 858-862, 1994.

20 Shimura M, Saito A, Matsuyama S, Sakuma T, Terui Y, Ueno K, Yumoto H, Yamauchi K, Yamamura K, Mimura H, Sano Y, Yabashi M, Tamasaku K, Nishio K, Nishino Y, Endo K, Hatake $\mathrm{K}$, Mori Y, Ishizaka Y and Ishikawa T: Element array by scanning $\mathrm{X}$-ray fluorescence microscopy after cis-diamminedichloroplatinum(II) treatment. Cancer Res 65: 4998-5002, 2005.

21 Elferink F, van der Vijgh WJ, Klein I and Pinedo HM: Interaction of cisplatin and carboplatin with sodium thiosulfate: reaction rates and protein binding. Clin Chem 32: 641-645, 1986.

22 Goel R, Cleary SM, Horton C, Kirmani S, Abramson I, Kelly C and Howell SB: Effect of sodium thiosulfate on the pharmacokinetics and toxicity of cisplatin. J Natl Cancer Inst 81: 1552-1560, 1989.

Received September 7, 2018

Revised September 22, 2018

Accepted September 28, 2018 